\title{
Hartree-Fock calculations of a finite inhomogeneous quantum wire
}

\section{Citation}

Qian, Jiang and Bertrand I Halperin. 2008. Hartree-Fock calculations of a finite inhomogeneous quantum wire. Physical Review B 77(8): 085314.

\section{Published Version}

http://dx.doi.org/10.1103/PhysRevB.77.085314

\section{Permanent link}

http://nrs.harvard.edu/urn-3:HUL.InstRepos:2887035

\section{Terms of Use}

This article was downloaded from Harvard University's DASH repository, and is made available under the terms and conditions applicable to Other Posted Material, as set forth at http:// nrs.harvard.edu/urn-3:HUL.InstRepos:dash.current.terms-of-use\#LAA

\section{Share Your Story}

The Harvard community has made this article openly available.

Please share how this access benefits you. Submit a story.

Accessibility 


\title{
Hartree-Fock calculations of a finite inhomogeneous quantum wire
}

\author{
Jiang Qian and Bertrand I. Halperin \\ Lyman Laboratory of Physics, Harvard University, Cambridge, Massachusetts 02138, USA
}

(Received 20 July 2007; published 21 February 2008)

\begin{abstract}
We use the Hartree-Fock method to study an interacting one-dimensional electron system on a finite wire, partially depleted at the center by a smooth potential barrier. A uniform $1 \mathrm{~T}$ Zeeman field is applied throughout the system. We find that with the increase in the potential barrier, the low density electrons under it go from a nonmagnetic state to an antiferromagnetic state and then to a state with a well-localized spin-aligned region isolated by two antiferromagnetic regions from the high density leads. At this final stage, in response to a continuously increasing barrier potential, the system undergoes a series of abrupt density changes, corresponding to the successive expulsion of a single electron from the spin-aligned region under the barrier. Motivated by the recent momentum-resolved tunneling experiments in a parallel wire geometry, we also compute the momentum-resolved tunneling matrix elements. Our calculations suggest that the eigenstates being expelled are spatially localized, consistent with the experimental observations. However, additional mechanisms are needed to account for the experimentally observed large spectral weight near $k=0$ in the tunneling matrix elements.
\end{abstract}

DOI: 10.1103/PhysRevB.77.085314

PACS number(s): 73.21.Hb, 73.21.- b, 73.23.Hk

\section{INTRODUCTION}

One-dimensional (1D) electronic systems have been proven to be a very fruitful field in the studies of interacting many-body systems. The infinite homogeneous onedimensional electron system has been extensively studied. At high density $n \gg a_{B}^{-1}$, where $a_{B}=\epsilon \hbar^{2} / m e^{2}$ is the Bohr radius, the low energy physics of the system is described well by the Luttinger model, ${ }^{1}$ with spatially extended electronic states as well as separate spin and charge excitations propagating at different speeds $v_{s}$ and $v_{c}{ }^{2}$ At low density $n \ll a_{B}^{-1}$, a system with a long-range interaction can be described best as a fluctuating Wigner crystal, with electrons being confined around their equilibrium positions by their mutual repulsion, though quantum fluctuations prevent a true long-range order. The excitations in this case are the density fluctuations (plasmon) of the Wigner crystal and the spinon excitation from the Heisenberg antiferromagnetic spin chain created by the exchange of the neighboring localized electrons through a barrier formed by their mutual repulsions. ${ }^{3}$ For a system with a short-range interaction, at low densities $n \ll d^{-1}$, where $d$ is the range of the interaction, the charge sector can be described as a weakly interacting gas of spinless fermions, and the spin sector can again be described as a Heisenberg antiferromagnetic chain with an appropriate exchange constant $J$.

Theoretical analyses have also considered the case of a finite wire, with either sharp or soft confinement at the ends. ${ }^{4-6}$ By contrast, the case of a spatially inhomogeneous system, with a low density region in the center of the wire, has not been extensively explored. In recent experiments, Steinberg and co-workers ${ }^{7,8}$ used a negatively charged metal gate to partially deplete the central region of a finite quasione-dimensional wire and studied the low density region by means of momentum-conserved tunneling from a parallel "semi-infinite" wire with higher electron density. They found a striking transition from a regime of extended electronic states to a regime of apparently localized states, as the elec- tron density at the center of the wire is lowered by the negative gate voltage. At a critical value, the electrons at the Fermi level seem to change abruptly from an extended state with well-defined momentum into a localized state with a wide range of momentum components. In the extended state regime, the tunneling measurements show a smooth variation of the electron density in the wire as a function of the gate voltage. In contrast, in the localized regime, the tunneling only occurs at a series of discrete resonant gate voltages, corresponding to the successive expulsion of a single electron from a Coulomb blockaded region that is somehow formed under the repulsive gate. Transport measurements show that the electrical conductance along the wire is much smaller than $e^{2} / h$ when the electron-density under the center gate is low enough to be in the localized regime. Furthermore, measurements of momentum-conserved tunneling from a second parallel wire show a dramatic change in behavior in the localized regime, as we shall discuss further below.

Motivated by the above experiments, we have turned to the Hartree-Fock method to investigate the physical properties of a system of interacting electrons on a finite wire with a barrier potential at its center, with a special focus on the evolution of the low density electrons.

In previous work, Matveev ${ }^{3}$ studied the case of transport properties of 1D interacting electrons through an adiabatic barrier and concluded that the conductance is $2 e^{2} / h$ at low temperature and $e^{2} / h$ at high temperature. ${ }^{9}$ However, he did not explore the regime where the electron density under the barrier is nearly depleted and the two terminal conductance becomes much smaller than $e^{2} / h$.

Mueller $^{6}$ explored the crossover from the nonmagnetic state to the Wigner crystal antiferromagnetic state when reducing the electronic density in a finite wire using a restricted Hartree-Fock method. He mostly considered a finite wire that is relatively uniform in the center region, under no external magnetic field. In Appendix B of his paper, he briefly considered a wire with an additional potential barrier in the center and found a low density Wigner-crystal-like 
regime under the barrier. He did not investigate further the density and spin evolution of his system as the density under the barrier is further depleted nor did he study the momentum-dependent tunneling amplitude in the case with a low density center region.

Meir and co-workers ${ }^{10,11}$ studied the formation of magnetic moments in a quantum point contact (QPC) in a twodimensional geometry using spin-density-functional. They found that as the density inside QPC rises above pinch-off, a magnetic moment forms inside the opening channel. In longer QPC, the magnetic moments take the form of an antiferromagnetically ordered chain. The conducting channels inside the QPC can be roughly modeled as a onedimensional system with a smooth potential barrier, and the antiferromagnetic order under the barrier they found is consistent with one of the magnetic phases we found in our study. However, unlike the QPC system, our strictly onedimensional system in a strong magnetic field further becomes ferromagnetic in low density region near depletion. Furthermore, in our model, we use a modified form of electron interaction to take into account the screening in the tunneling experiments described below.

The paper is organized as follows. In Sec. II, we introduce our basic model, our choice of parameters, and the numerical method we employed. In Sec. III, we present the physical picture of successive magnetic phases our system goes through as we increase the potential barrier and the crossovers between phases. In Sec. IV, we make a more detailed analysis of the form of the wave function at Fermi energy near depletion. Motivated by the experimental measurements by Steinberg and co-workers, ${ }^{7,8}$ we also compute the momentum dependence of the tunneling matrix elements for our system. In Sec. V, we compare our results to the HartreeFock calculation for an infinite homogeneous system and a noninteracting inhomogeneous system and discuss the implications of the electrical conductance measurement from our calculation. In Sec. VI, we summarize our results and their comparisons with experiments.

\section{MODEL}

We consider a system of one-dimensional interacting electrons in a wire of length $L=6 \mu \mathrm{m}$ with periodic boundary conditions. A uniform magnetic field $B$ is applied throughout the system, which couples only to the spins in our model and which, in most of our calculations, we set to $1 \mathrm{~T}$. In the experiments, ${ }^{7,8}$ a magnetic field of $1-3 \mathrm{~T}$ was typically applied.

With the above-mentioned experiments in mind, we assume that the electrons interact via a Coulomb potential with both a short-range and a long-range cutoff. The short-range cutoff comes from the finite width of the experimental wire. We model it by simply modifying a $1 / z$ potential to $1 / \sqrt{z^{2}+W^{2}}$, where $z=x-x^{\prime}$ is the separation of two electrons along the wire and $W$ is the short-range cutoff, roughly on the order of half of the width of the wire. This densityindependent short-range cutoff is appropriate in our case of a sharp confinement transverse to the direction of the wire formed by the cleaved edge overgrowth. The long-range cut-

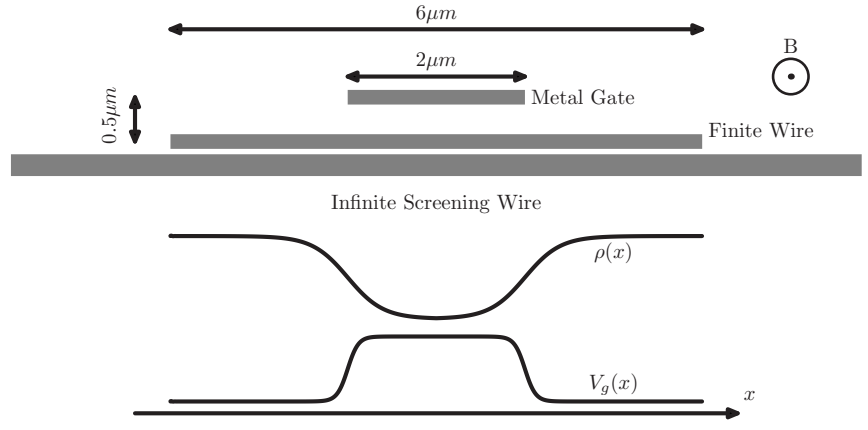

FIG. 1. A schematic illustration of the geometric configurations and the potential and density profiles considered in this paper.

off is the result of the screening effect from the higher density wire parallel to the short wire in the tunneling experiment. We model it by putting a second wire, which is simplified to be infinitely long and perfectly conducting, parallel to the finite wire under study, at a center to center distance $d$, as shown in Fig. 1. The resulting form of the interaction $U(z)$ can be easily derived, as discussed by Fiete $e t$ $a l .{ }^{5}$ At short distance $x \ll W, U$ levels off smoothly as $1 / \sqrt{z^{2}+W^{2}}$, whereas at long distance $x \gg d$, it decays much more rapidly than the Coulomb potential $1 / z$. Following the experimental setup, in this paper, we choose $W=0.01 \mu \mathrm{m}$ and $d=0.031 \mu \mathrm{m}$ and choose the strength of the Coulomb interaction to correspond to the value in the bulk GaAs, yielding a Bohr radius $a_{B} \approx 0.01 \mu \mathrm{m}$.

In the experiments, a negatively charged $2 \mu \mathrm{m}$ long metal gate at $0.5 \mu \mathrm{m}$ above the finite wire is used to reduce the density at the center region of the wire, as illustrated in Fig. 1. To approximate the effect of the gate, we use a smooth bare barrier potential of the form

$$
V_{G}(x)=\frac{V_{g}}{1+\exp \left[\left(|x|-L_{g} / 2\right) / L_{s}\right]} .
$$

Here, $L_{g}$ is the length of the potential barrier, which we choose to be the length of the experimental metal gate, $2 \mu \mathrm{m}$, in our calculation. $L_{s}$ controls the sharpness of the edge of the potential barrier, which we choose to be on the order of $0.5 \mu \mathrm{m}$, the distance from the gate to the finite wire. The quantity $V_{g}$ will be referred to below as "gate voltage," although it is actually only proportional to (minus) the applied voltage $V_{G}$. The normalization is such that $V_{g}$ is the bare potential at the center of the barrier region. The spatial form of this potential can be seen in Fig. 2.

In the experiments, more than one transverse mode in the quantum wires can be occupied. Correspondingly, in the wire of our model, we maintain two separate subbands of electrons, which we assume to interact only through the Hartree term to the electrons in the other subband. This is equivalent to the assumption that in the experimental wire, one can ignore any effects of scattering or exchange between electrons in different transverse modes. In our model, an energy difference of $42 \mathrm{meV}$ separates the bottom of the two subbands, corresponding to the energy separation of the lowest two transverse modes in a square well of width of $0.02 \mu \mathrm{m}$. 


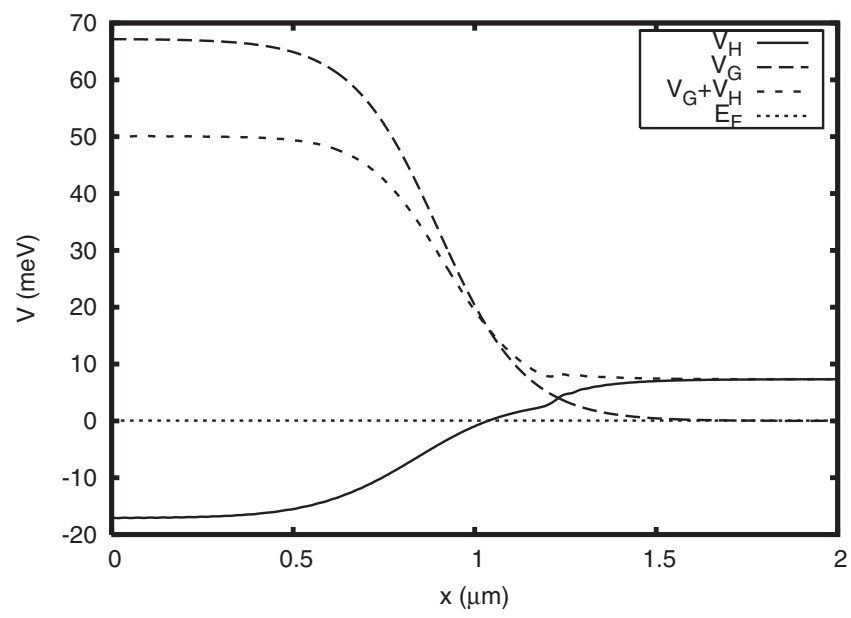

FIG. 2. The shape of the bare potential barrier $V_{G}$, the Hartree potential $V_{H}$, and their sum at gate voltage $V_{g}=67.2 \mathrm{meV}$. The density distribution at this gate voltage is shown at the bottom of Fig. 4. All potentials are symmetric around $x=0$, and only the center part $x<2 \mu \mathrm{m}$ is shown. The Fermi energy $E_{F}$ of the electrons is also shown.

In summary, our Hartree-Fock Hamiltonian can be written as

$$
\begin{aligned}
H \psi_{\sigma b}(x)= & -\frac{\hbar^{2}}{2 m^{*}} \frac{\partial^{2} \psi_{\sigma b}(x)}{\partial x^{2}} \\
& +\left[V_{G}(x)+\Delta_{b}-\frac{1}{2} g^{*} \mu_{B} B \sigma_{z}\right] \psi_{\sigma b}(x) \\
& +V_{H}(x) \psi_{\sigma b}(x)-\int d x^{\prime} V_{F}^{\sigma b}\left(x, x^{\prime}\right) \psi_{\sigma b}\left(x^{\prime}\right), \\
V_{H}(x)= & \int d x^{\prime}\left[\sum_{i, \sigma^{\prime}, b^{\prime}}\left|\psi_{i \sigma^{\prime} b^{\prime}}\left(x^{\prime}\right)\right|^{2}\right] U\left(x-x^{\prime}\right), \\
V_{F}^{\sigma b} & =\sum_{i} \psi_{i \sigma b}(x) \psi_{i \sigma b}^{*}\left(x^{\prime}\right) U\left(x-x^{\prime}\right) .
\end{aligned}
$$

Here, $m^{*} \approx 0.067 m_{e}$ is the effective electron mass in bulk GaAs, $g^{*} \approx 0.44$ is the effective $g$ factor in bulk GaAs, and $\mu_{B}$ is the Bohr magneton. $\psi^{*}(x)$ is the complex conjugate of $\psi(x) . \sigma=(\uparrow, \downarrow)$ is the spin index. $b=0,1$ is the subband index: $\Delta_{0}=0 \mathrm{meV}$ for $\psi_{\sigma 0}$ in the first subband and $\Delta_{1}=42 \mathrm{meV}$ for $\psi_{\sigma 1}$ in the second subband. The summation over $i$ in computing the Hartree potential $V_{H}$ and Fock kernel $V_{F}$ is over all the occupied states in a specific spin and subband. Notice, as discussed in the previous paragraph, that in computing the Fock potential kernel $V_{F}^{\sigma b}$, we only sum over the occupied states with the same spin $\sigma$ and in the same subband $b$ as the eigenstate it is acting on.

The numerical method we use in our calculation is a restricted Hartree-Fock method. ${ }^{12}$ The electron spins are required to be either parallel or antiparallel to the applied magnetic field, so canted spin structures are not allowed. In the Appendix, however, we consider the effects of canting in an infinite homogeneous wire, and we argue that canting would

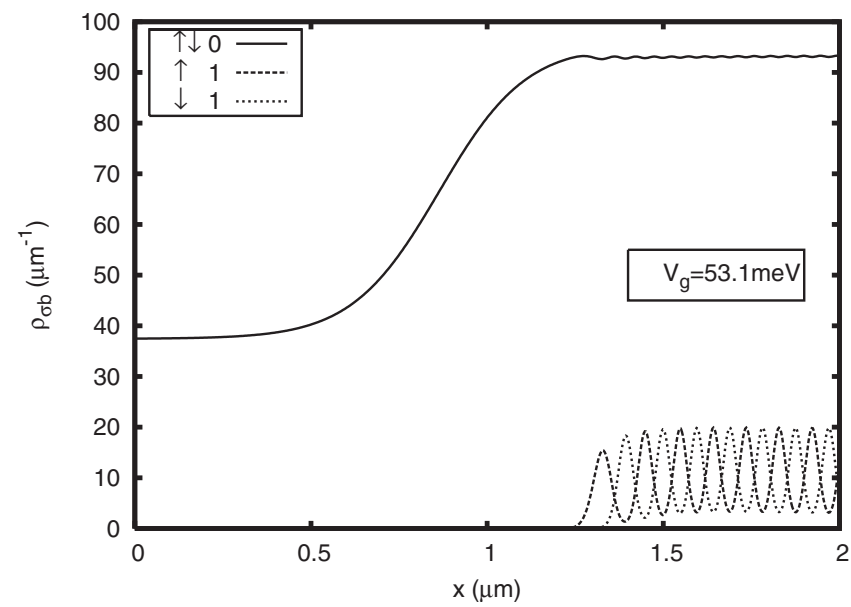

FIG. 3. The density distributions $\rho_{\sigma b}(x)$ for the different spin states and subbands in the ground state in a high density regime where the center region is nonmagnetic. Identifications of the indices $\sigma, b$ for the curves are shown in the inset. For the lower subband $(b=0)$, the densities of the two spin states are indistinguishable and are shown by a single curve. All densities are symmetric around $x$ $=0$, and only the center part $x<2 \mu \mathrm{m}$ is shown.

have a negligible effect on results for the inhomogeneous system for the parameters of interest to us.

Starting from solutions to the noninteracting potential barrier problem, we iteratively use the Hartree-Fock method until the convergence between iterations is achieved. Throughout the calculation, we fix the total number of electrons in the finite wire to be $N=1000$, whereas the occupation numbers in each spin or subband species remain free to change.

In this paper, we focus on the depletion of the first subband under the barrier. In this regime, the second subband is fully depleted under the barrier and is only occupied in the outer regions where the total density is high. Consequently, in our calculations, the second subband serves mostly as a reservoir for the electrons under the barrier.

\section{MAGNETIC PHASES}

At high densities, we find that the first subband under the gate is essentially unpolarized, as shown in Fig. 3. At an electron density $\rho=40-50 \mu \mathrm{m}^{-1}$, or $\rho_{\sigma 0}=20-25 \mu \mathrm{m}^{-1}$ per spin, an antiferromagnetic order emerges at the low density region under the barrier, as shown in Fig. 4. The antiferromagnetic order parameter, the staggered magnetization $\tilde{M}$, grows steadily as the density decreases with the increase of $V_{g}$. There is no sharp transition between the nonmagnetic and the antiferromagnetic solutions.

At $\rho=\rho^{*} \sim 20 \mu \mathrm{m}^{-1}$ under the barrier, a spin-aligned center region appears and rapidly expands, as shown in Fig. 5. As seen in the figure, the spin-aligned region at the center is sandwiched by two strongly antiferromagnetic regions on each side. Within a narrow range of $V_{g}$, the spin-aligned region expands to a maximum length, containing $N_{f}=10$ electrons, as shown in the bottom of Fig. 5. From that point on to its full depletion, the center spin-aligned region undergoes a series of transitions, each representing the expulsion 


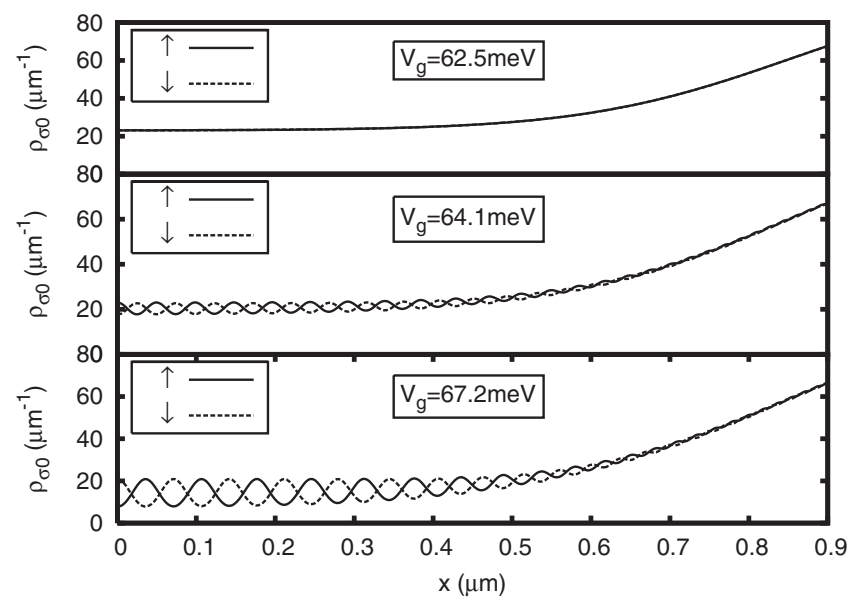

FIG. 4. Densities of spin-up and spin-down electrons in the center region showing the emergence of antiferromagnetic order with increasing $V_{g}$. All densities are symmetric around $x=0$, and only the center part of the first-subband electron densities $x<0.9 \mu \mathrm{m}$ is shown.

of one electron from the spin-aligned region. In contrast with the nonmagnetic and antiferromagnetic regimes, where the electronic density $\rho(x)$ under the barrier changes smoothly with $V_{g}$, here, the $\rho(x)$ in the spin-aligned center region of the barrier varies discontinuously with an increase in $V_{g}$. Figures 6-8 show the details of one of such transitions, with the number of electrons in the spin-aligned region changing from $N_{f}=8$ to $N_{f}=7$ at $V_{g}^{*} \approx 71.75 \mathrm{meV}$. In Fig. 6, we see a crossover in the total energy of the Hartree-Fock ground state $E$, where the $N_{f}=8$ solution has a lower energy for $V_{g}<V_{g}^{*}$ and the $N_{f}=7$ solution becomes the ground state for $V_{g}>V_{g}^{*}$. In terms of the occupation numbers of the states of different subbands and spins, this transition corresponds to the expulsion of one electron from the spin-up first subband to the spin-up second subband.

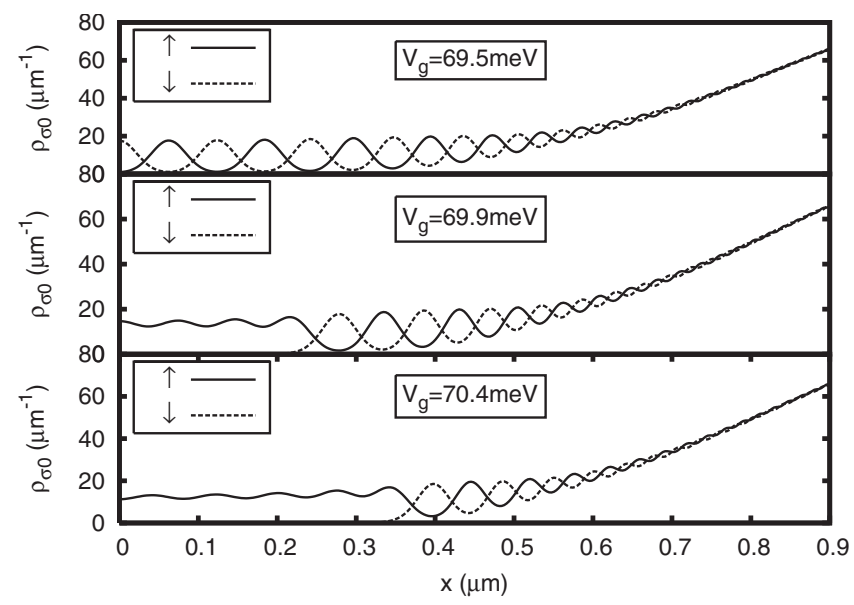

FIG. 5. The transition from antiferromagnetic order to spin aligned at the center region in the form of a spin-aligned region which expands rapidly from the center. All densities are symmetric around $x=0$, and only the center part of the first-subband electron densities $x<0.9 \mu \mathrm{m}$ is shown.

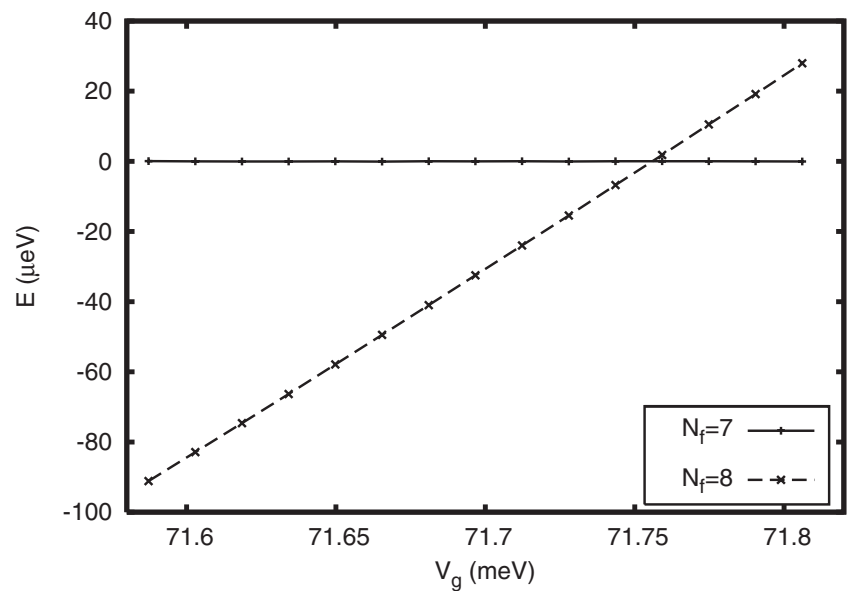

FIG. 6. This plot shows the crossover as a function of the barrier height $V_{g}$ of the ground state energy $E$ of two Hartree-Fock solutions, labeled by the number of electrons $N_{f}$ in spin-aligned region at the center. For the sake of clarity, a quadratic function of $V_{g}$ is subtracted from each of the ground state.

In general, as $V_{g}$ increases, the transitions in our calculation always involve an expulsion of one spin-up firstsubband electron to the second subband outside the center region. However, spin flip transitions also happen. There are transitions showing the spin-down second subband absorbing the expelled electron from the spin-up first subband. In Fig. 7 , the detailed density changes in one transition are shown clearly. The spin-aligned region in each of the solutions is isolated quite well by the antiferromagnetic regions on its sides. The first-subband spin-down electron density $\rho_{\downarrow 0}$, which is not shown in Fig. 7 for the sake of clarity, drops steeply to zero for $|x| \leqslant 0.45 \mu \mathrm{m}$, where $\rho_{\uparrow 0}$ rises sharply, on both sides of the transition. The short antiferromagnetic re-

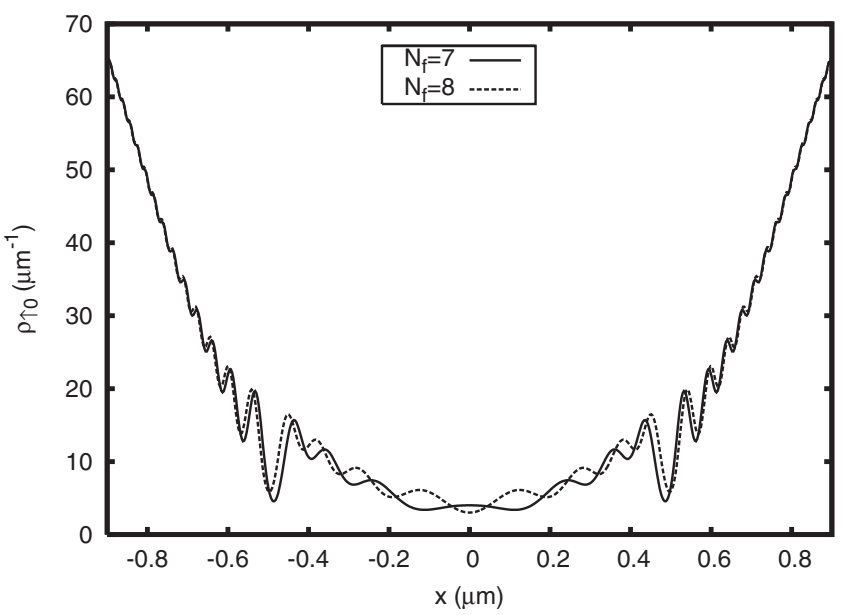

FIG. 7. Electronic density under the barrier before and after the transition shown in Fig. 6. Only the densities of first-subband spin-up electrons are plotted since electron densities in other states do not change greatly during the transition. The spin density labeled $N_{f}=8$ is taken at $V_{g}=71.74 \mathrm{meV}$ right before the crossover in Fig. 6 , and the one labeled $N_{f}=7$ is taken at $V_{g}=71.76 \mathrm{meV}$ right after it. 


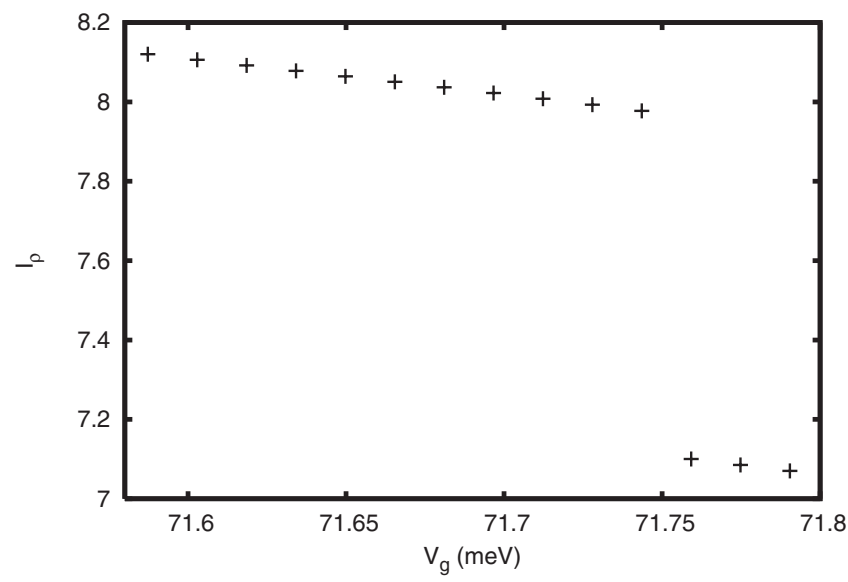

FIG. 8. $I_{\rho}$ is the integrated number of spin-up electrons in the lower band within the center region defined by $-x_{0} \leqslant x \leqslant x_{0}$, where $x_{0}=0.498 \mu \mathrm{m}$. As a function of the gate voltage $V_{g}$, we follow the lowest energy solution across the crossover shown in Fig. 6, i.e., the $I_{\rho}$ on the left of the crossover is computed from the solution $N_{f}$ $=8$ and the $I_{\rho}$ on the right from $N_{f}=7$. The drop in $I_{\rho}$ represents the expulsion of approximately 0.88 electron from within the center region.

gions on the two sides are only slightly shifted in the transition, whereas the center spin-aligned region undergoes the significant change from having eight peaks to having seven. By plotting the integrated density in the spin-aligned region, Fig. 8 shows that this change in $\rho_{\uparrow 0}$ indeed amounts to the expulsion of almost a whole electron from the region. The slight deficiency from unity is due to the slightly changed length of the spin-aligned region and possibly some small residual density from the spin-aligned states extending into the antiferromagnetic sides.

It may be possible to detect the existence of the central spin-aligned region by the application of a magnetic field $B_{\|}$ parallel to the quantum wire in question. For a state with $N$ spin-aligned electrons in the center region, the magnetic field will result in a Zeeman energy shift $E_{Z}=N g{ }^{*} m u_{B} B_{T} / 2$, where $g^{*} \approx 0.44$ is the effective $g$ factor in bulk GaAs, $m u_{B}$ is the Bohr magneton, and $B_{T}=\sqrt{B_{\|}^{2}+B^{2}}$ is the strength of the total magnetic field. Due to this energy shift, the transition voltage from the $N$ to the $N+1$ spin-aligned electron state will be shifted to a larger value because the $N+1$ electron state energy will be lowered by $\Delta E_{Z}=g^{*} m u_{B} / 2$ relative to the $N$-electron state. Such a shift may be detectable for a large change in the combined field $B$. For example, in the $N=8$ to $N=7$ transition discussed above, a change $\delta B_{T}=4 \mathrm{~T}$ in the combined field will result in a shift of $\Delta E_{Z} \approx 0.092 \mathrm{meV}$. This is about one-fifth of the typical spacing between transition gate voltage $V_{g}^{*}$, which is approximately $0.45 \mathrm{meV}$.

\section{WAVE FUNCTIONS AND MOMENTUM-CONSERVED TUNNELING}

In the momentum-conserved tunneling experiments of Steinberg et al., electrons tunnel between the finite wire and a parallel "infinite" wire while conserving their momentum

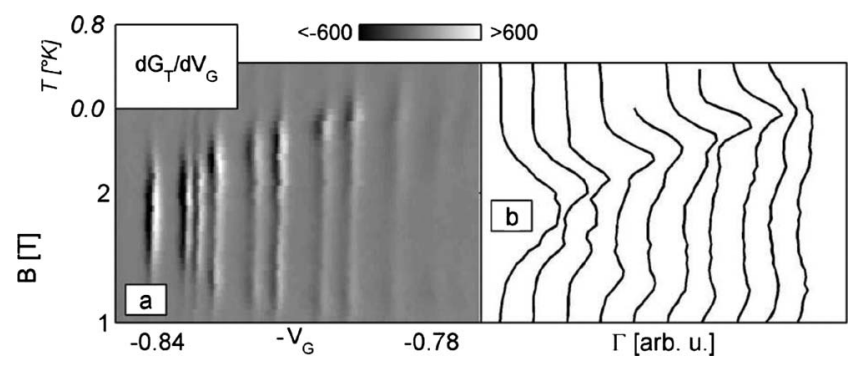

FIG. 9. High resolution measurement of the localized features from Steinberg et al. (Ref. 8). On the left is the raw tunneling data $d G / d V_{G}$. On the right are the corresponding tunneling rates $\Gamma(B)$, extracted from the fitting of the localized features to Coulomb blockade line shape at each magnetic field. $\Gamma(B)$ is proportional to $|M(k)|^{2}$, where $M$ is defined in Eq. (5) and $B$ controls the momentum of the tunneling electron.

in the wire direction. A magnetic field $B$ perpendicular to the cleaved edges defining the two quantum wires gives a controllable momentum boost $q_{B}=e B d / \hbar$ to the electrons tunneling between the wires, where $d$ is the distance between the wires. At low temperature and small source-drain bias, the tunneling conductance $G_{T}\left(B, V_{g}\right) \propto|M|^{2}$, where the matrix element $M$ has the following physical interpretation: ${ }^{4,5}$

$$
M=\int_{-\infty}^{\infty} d x e^{-i k x} \Psi_{\mathrm{eff}}^{N}(x),
$$

where we have defined the "quasi-wave-function" as

$$
\Psi_{\mathrm{eff}}^{N} \equiv\langle N-1|\psi(x)| N\rangle,
$$

with $|N\rangle$ being the $N$-electron many-body ground state in the finite wire, $\psi(x)$ being the electron annihilation operator at position $x$ in the finite wire, and $k=q_{B} \pm k_{F}$, where $k_{F}$ is the Fermi wave vector in the infinite wire. (We neglect here electron-electron interactions in the infinite wire.) The momentum dependence of $|M|$ can be extracted from the magnetic-field dependence of the Coulomb blockade peak. ${ }^{8}$ In the localized regime, expulsion of an electron from the region under the gate is signaled by a vertical stripe in a color plot of the tunnel conductance in the plane of gatevoltage $V_{G}$ and magnetic field $B$ (see the left panel of Fig. 9). The momentum dependence of $|M(k)|^{2}$ is obtained by integrating the intensity across a given vertical stripe, at a fixed value of the magnetic field, and comparing the results for different values of $B$. As seen in the right panel of Fig. 9, except for the last peak, the momentum dependence of the $|M(k)|^{2}$, found in the experiments, typically shows to two wide peaks, as well as a broad momentum distribution between the peaks, signaling relatively localized wave functions.

In our calculation, the $N-1$ and $N$-electron states should be the complete Slater determinants of the eigenstates of the corresponding Hartree-Fock Hamiltonians. As a simplifying approximation, we may assume that after expelling one electron, the rest of the eigenstates are not affected. In this case, $\Psi_{\text {eff }}^{N}$ is simply the wave function of the electron being expelled and $M(k)$ is its Fourier transform. $|M(k)|^{2}$ computed 


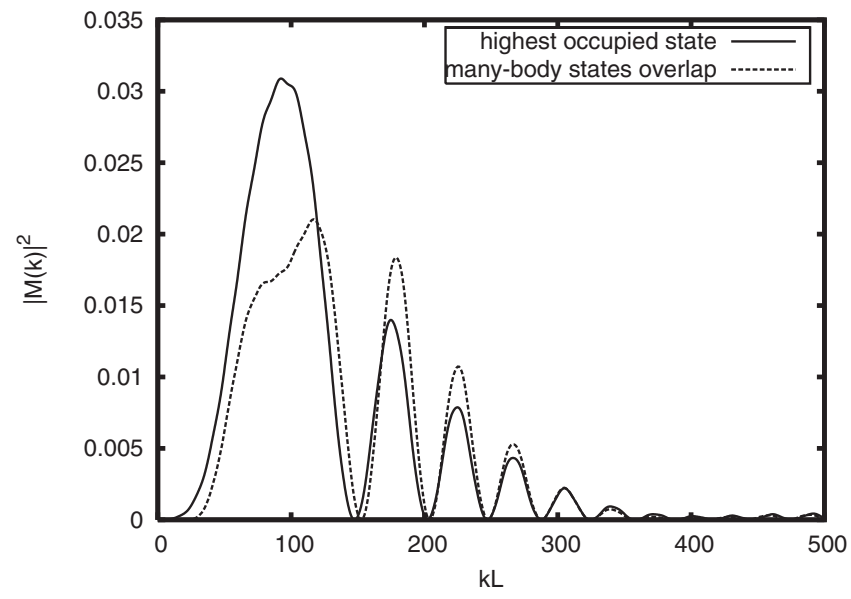

FIG. 10. $|M(k)|^{2}$ for the transition shown in Fig. 6 from $N_{f}=8$ to $N_{f}=7$. The solid line is the squared Fourier transform of the wave function being expelled. The dotted line is computed from the overlap between full Slater determinants.

this way for the transition $N_{f}=8$ to $N_{f}=7$ is shown as the solid line in Fig. 10. The dashed curve is the result obtained by using the full Slater determinants of the $N-1$ and $N$-electron solutions to compute the matrix elements. This shows an orthogonality-catastrophe-type reduction to the overall spectral density weight. Both of the calculated matrix elements show a relatively broad momentum distribution, consistent with the experimental result. However, the experimental result shows heavy spectral weight near $k=0$, between the two largest peaks at $k= \pm k_{\max }$, and little weight outside them, whereas our calculations show little weight between the largest peaks and considerable weight outside, see Fig. 10. This discrepancy suggests that additional mechanisms are needed to explain the finer details of the observed momentum distribution $|M(k)|^{2}$.

Insight into the calculated shape of $M(k)$ can be gained by looking at the wave function in position space for the electron being expelled. Figure 11 shows the wave function in position space, for which the squared Fourier transform is the solid curve in Fig. 10. We see that the wave function has relatively large weight in the central spin-aligned region, roughly for $|x|<0.5 \mu \mathrm{m}$. However, it also has significant weight outside the barrier and in the transition region between. As will be discussed further below, the wave function is qualitatively similar to what one would expect in the WKB approximation for a state slightly above the top of a smooth potential barrier. The separation between successive zeros of the wave function is largest near the center, where the amplitude is largest, and it decreases monotonically in the transition region, where the amplitude decreases gradually and the particle velocity increases. The Fourier transform $M(k)$ has its largest amplitude at a value $|k|=k_{m}$ corresponding to the spacing between zeros in the center region and has additional weight at larger wave vectors, corresponding to the smaller spacing of zeros in the transition region. By contrast, the experimental results look like what one would find for a particle confined in a soft potential well, where the zeros of the wave function would be closest together near the center of the well and be farther apart at the two ends. In this case,

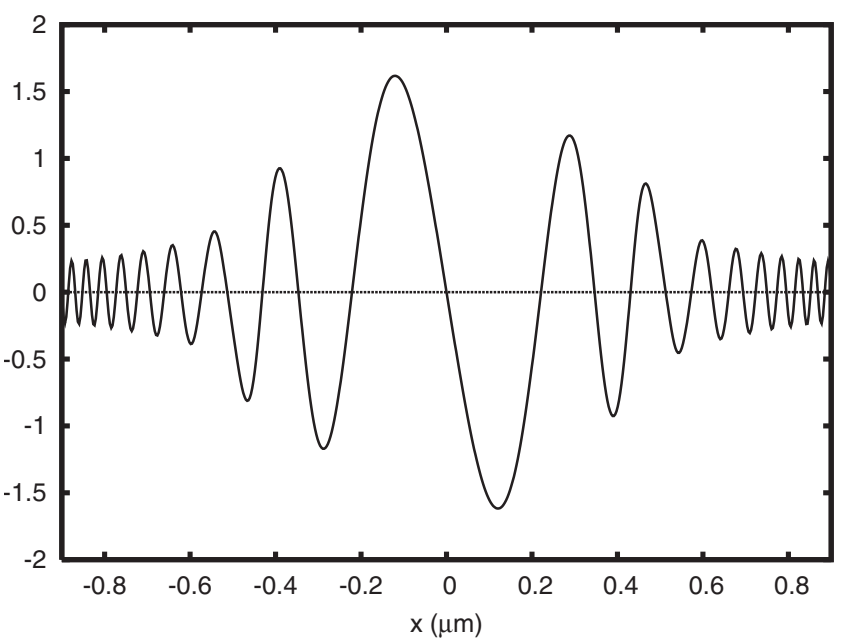

FIG. 11. This is the highest occupied eigenstate of the solution at $V_{g}=71.74 \mathrm{meV}$ right before the crossover in Fig. 6. The wave function is normalized and only the center region is plotted.

the Fourier transformed wave functions have significant weight for $|k|$ larger than the peak value $k_{m}$ but very little weight at larger $|k| .^{4,5}$ In either case, one finds zeros in $M(k)$ and oscillations in the amplitude, arising from interference between contributions at spatial points $x$ and $-x$.

\section{DISCUSSION}

\section{A. Hartree-Fock for a homogeneous system}

In order to better understand the results of our calculations, it will be helpful to recall some features of HartreeFock calculations for an infinite homogeneous onedimensional electron system. At high electron densities, where electron-electron interactions are relatively weak, with no applied magnetic field, one finds a nearly-free electron gas, with a small gap at the Fermi energy, caused by a spindensity wave corresponding to the wave vector $Q=2 k_{F}$. In position space, this means that the unit cell contains precisely two electrons on average, with a weak polarization, alternating between spin up and spin down, along a quantization axis that has arbitrary direction. ${ }^{13}$ The total charge density will have a period half that of the spin density, i.e., there is just one electron in each charge period. The amplitude of the charge modulation will be proportional to the square of the spin-density amplitude, when these modulations are small. For pure Coulomb interactions, the amplitude of the spin-density modulation will fall off rapidly at high densities $\rho$, roughly as $\exp (-a \rho)$, where $a$ is the length of the order of the Bohr radius. If the electron-electron interaction is smooth at short distances, the amplitude of the spindensity modulation can still fall off faster with increasing $\rho$. For a system of finite length $L$, we would generally not expect to find any spin-density modulation if the antiferromagnetic coherence length, which is inversely proportional to spin-density amplitude of the infinite system, becomes larger than $L$.

As the electron density is lowered, the amplitudes of the spin and charge modulations both grow until one reaches the 
situation of a Wigner crystal, where there is strong modulation in the charge density and there is nearly complete spin polarization, alternating up and down for successive electrons. At still lower densities, the Hartree-Fock approximation predicts a phase transition to a fully aligned ferromagnetic state. The ferromagnetic state is, of course, an artifact of the Hartree-Fock approximation, as it is known ${ }^{14}$ that the exact ground state is a spin singlet for $B=0$. However, a state of full spin alignment should occur at low densities for $B \neq 0$, and the Hartree-Fock approximation may be a reasonable description of this transition for $B=1 \mathrm{~T}$.

The predicted antiferromagnetic order is also an artifact of the Hartree-Fock approximation, as quantum fluctuations would be expected to replace the long-range spin order with correlations that fall off as a power of the distance. In an infinite system, charge density modulations will also be destroyed by quantum fluctuations of the positions of the electrons on the Wigner crystal. However, these quantum fluctuations can be relatively weak when there is strong repulsion between the electrons, and a significant charge density modulation may exist in a finite system of moderate length.

Hartree-Fock calculations for an infinite system, with the same interaction potential used in our finite system, are presented in the Appendix. We also discuss there the effect of spin canting in an applied magnetic field.

According to Fig. 13 (see Appendix), the Hartree-Fock transition to a fully spin-polarized state should occur at a density of approximately 16 electrons per micron for an infinite system with the parameters of the model under consideration in a Zeeman field $B=1 \mathrm{~T}$. This is similar to the density $\rho^{*} \approx 20 \mathrm{~nm}^{-1}$, where we found the onset of a center region with full spin alignment in our calculations for the system with a barrier.

\section{B. Inhomogeneous system without interactions}

It is also useful to review what one would expect for an inhomogeneous system analogous to the wire under consideration but without electron-electron interactions. In particular, we may consider what should happen as one varies the height of a smooth center barrier, similar to the bare potential barrier in Fig. 2 or to the self-consistent potential in that figure, including the Hartree potential but not the nonlocal exchange potential. By comparing this qualitative picture with the results of our Hartree-Fock calculations, we can see better whether there are features of the latter which reflect in an essential way the exchange and correlation features of a strongly interacting many-electron system.

It is important to note that the total length of our system is finite, so there will be a discrete set of energy levels for the system as a whole. As the total length is $6 \mu \mathrm{m}$, and the flat potential area under the gate is of order of $1-2 \mu \mathrm{m}$, the majority of the length is outside the barrier region. The density of states in a noninteracting one-dimensional system is inversely proportional to the electron density $\rho$; however, the local density of states for electrons in the barrier region can be much higher than the density of states outside if the electron density is sufficiently low in the barrier region. Thus, if the chemical potential is fixed and the height of the barrier is lowered below the Fermi energy, we may expect to see a closely spaced sequence of electrons entering into states whose amplitudes are highly concentrated in the center region. Indeed, if the overall system length is not too large, we may expect a large fraction of the probability density for each added electron to be located in the barrier region.

It should be noted that the upper wire in the experiments of Ref. 8 has an overall length that is not very different from that of the wire used in our calculations. The experimental wire is not truly isolated but is tunnel coupled to leads in its outer regions; so its energy levels should actually be lifetime broadened. If the escape rate from the wire is smaller than the spacing between energy levels, however, the features of a finite system should be maintained.

For noninteracting electrons, if the total number of particles is fixed (rather than the chemical potential), then the electrons entering the center must be transferred from electron states outside the barrier region (e.g., states belonging to a second subband), and the Fermi level will itself decrease each time an electron is added to the center region. If the level spacing of the outside bands is larger than the level spacing of the center region, then the spacing between gate potentials where successive electrons enter the center region will be determined by the larger energy spacing between these reservoir states.

We may also consider what would happen if one had an infinite system of noninteracting electrons, with a flat barrier of finite length in the center. Suppose the potential $V(x)$ is zero outside the barrier region and equal to $V_{g}$ at the center of the barrier. If the barrier is smooth enough so that one can use the WKB approximation, then the wave function $\psi(x)$ for a state with energy $E$ slightly above $V_{g}$ will have an amplitude which is larger inside the central region than that outside by a factor $\{[E-V(x)] / E\}^{-1 / 4} \approx\left[\rho_{0} / \rho(x)\right]^{1 / 2}$, where $\rho(x)$ is the cumulative (Thomas-Fermi) electron density at point $x$ from all states with energy less than $E$, and $\rho_{0}$ is the electron density far from the barrier. For a smooth barrier, we see that $\rho(x)$, and hence the amplitude of the wave function $\psi$, should have a maximum in the center of the barrier and fall off monotonically with increasing $|x|$. The spacing between successive zeros of $\psi$ should be given by $1 / \rho(x)$, which should decrease monotonically with increasing $|x|$. We note that the Hartree-Fock wave function plotted in Fig. 11 is qualitatively consistent with these features.

If the barrier height is varied continuously at fixed Fermi energy, for an infinite system, when the WKB approximation is valid, the wave functions will vary continuously, and the number of particles above the barrier will likewise vary in a continuous fashion The WKB approximation will break down, however, if the energy $E$ gets too close to the top of the barrier. For a smooth potential, one expects the WKB approximation to be valid for all but the last one or two states above the barrier. By contrast, for a flat-topped potential that falls off relatively abruptly at the ends of the barrier, deviations may be more pronounced. In this case, we may find a number of resonant states above the barrier, which have very small electron density outside the barrier region and which exist only in narrow energy bands. Then, as the 


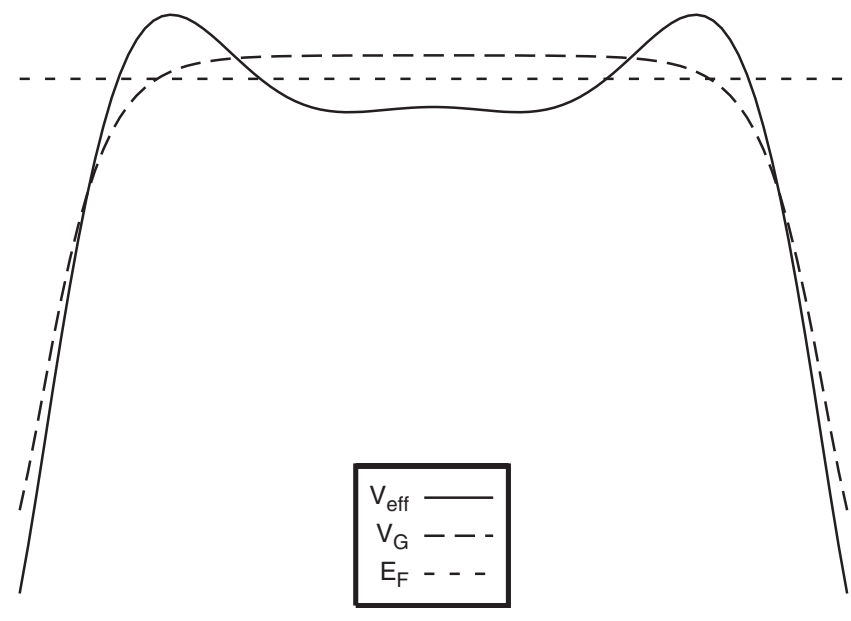

FIG. 12. A schematic plot of an effective barrier potential $V_{\text {eff }}$ that could produce a form of the wave function more consistent with the results of the tunneling experiments. The dashed line is the bare potential barrier $V_{G}$ and the dotted line is the Fermi level $E_{F}$.

gate voltage is varied, the number of electrons in the barrier region will increase by one in a narrow region of the gate voltage, as each resonance passes through the Fermi energy. In the limit of a potential $V(r)$ that drops sharply to $V=-\infty$ at the edge of the barrier, a wave function $\psi(x)$ with weight inside the barrier region will vanish outside this region; the wave functions and energy levels will be discrete and will be the same as if there was an infinitely high potential at the end of the barrier.

We may also imagine a situation where the self-consistent potential $V_{\text {eff }}(x)$ is smooth but nonmonotonic, having somehow developed a pair of maxima near the edges of the original barrier region, as illustrated in Fig. 12. In this case, there will be an energy range such that $E$ is smaller than the maximum value of $V_{\text {eff }}$ but larger than the value at the center of the barrier region. If this energy range is large enough, there may be a discrete series of states which are well localized in the classically allowed region, decay to a small value in the classically forbidden regions, and have only a small amplitude outside the barrier. The distance between zeros of the wave function will then increase with increasing $|x|$ in the region where the wave function is large. The form of $|M(k)|^{2}$ that one would obtain by taking the Fourier transform of this wave function will have a maximum intensity at a wave vector corresponding to the local Fermi wave vector $k_{F}$ at $x=0$ and will have significant weight for $|k|<k_{F}$ but very little weight at $|k|>k_{F}$. This result is qualitatively similar to the observations of Steinberg et al. (illustrated in Fig. 9). However, it is quite different from what we have obtained from our Hartree-Fock calculations (illustrated in Fig. 10), where there is considerable weight at large $k$.

The spin-density structures obtained in our Hartree-Fock calculations suggest that a nonmonotonic self-consistent potential (similar to that in Fig. 12) might in fact have been a qualitatively reasonable representation of the Hamiltonian seen by the electrons with the majority spin orientation. Because there is a fully polarized spin-down electron on either side of the central region of spin-up electrons and because there is a strong repulsion between electrons of opposite spin, one might expect that the spin-up electrons would see an effective potential maximum at the positions of the spindown electrons. The strong antiferromagnetic order just outside this region suggests that there might be, locally, an antiferromagnetic energy gap at the Fermi energy, so that the wave function of the highest energy filled state would decay further as it passes through this region. Our numerical results suggest, however, that these effects, if they are present, are not strong enough to produce the type of localization one might have hoped for.

The qualitative resemblance between the calculated Hartree-Fock wave function and the WKB form for states above a smooth potential barrier, discussed above, appears to persist down to densities where there are only one or two electrons left at the top of the barrier.

\section{Electrical conductance}

A striking feature of the experimental results is the occurrence of a sharp drop in the conductance through the finite wire in a regime where there were still several electrons in the region below the center gate. (Here, we refer to the two terminal conductance $G$ measured through contacts at two ends of the finite wire and not the tunneling conductance $G_{T}$ for current flowing between the finite wire and the semiinfinite wire.) For a one-dimensional system of noninteracting electrons, in a potential $V(x)$ that vanishes outside a central region, one can relate the electrical conductance, using the Landauer-Buttiker formula, to the transmission probability for an incident electron at the Fermi energy. For a potential barrier which is symmetric under reflection, the transmission probability, in turn, can be expressed in terms of the phase shifts for states of even and odd parities. In the Hartree-Fock approximation, however, this analysis is complicated by several factors. Although electron-electron interactions in the leads are relatively weak because of the high electron density there, they would still give rise to antiferromagnetic order in a lead of infinite length, as discussed above. Thus, in principle, there should always be an energy gap at the Fermi energy, and phase shifts cannot be defined. In practice, this should not be a serious problem for our system because the calculated energy gap is extremely small at high densities, and one could estimate the conductance from phase shifts at energies outside of the energy gap but still close to the Fermi energy.

A more significant problem arises from the fact that our computations use a system of finite length, and we have only a discrete set of energy levels. Analyzing these wave functions, we may obtain even-parity and odd-parity phase shifts at a discrete set of energies, but we do not obtain both even and odd phase shifts at a single energy. We can obtain some estimates of the phase shifts for an infinite system, however, by looking at the alternation between energy levels for even and odd numbered wave functions of a given spin and band index in the finite system.

For an energy high above the barrier, we expect that WKB is a good approximation, which means that an incident particle is transmitted with essentially no reflection, correspond- 
ing to a conductance of $e^{2} / h$ per spin. This means that there is no difference in the phase shifts for even and odd parities. In our model calculations, we assume periodic boundary conditions at the ends of the wire. Then, for a large but finite system, when there is negligible reflection at the barrier, we expect energy levels to occur in pairs, with even- and oddparity states that are nearly degenerate.

For an energy well below the barrier, where there is nearly total reflection, the even- and odd-parity phase shifts should differ by approximately $\pi / 2$. Then, with periodic boundary conditions, we expect energy levels to alternate between even- and odd-parity states, with nearly equal spacings between them.

The energy spacings we find in our Hartree-Fock calculations are in good agreement with these expectations provided the Fermi level is not too close to the barrier top. Thus, we have near perfect transmission when the Fermi level is well above the barrier and near perfect reflection when it is well below. However, we have not been able to analyze the conductivity in the most interesting region, when there are only a few electrons in the spin-polarized region at the top of the barrier, essentially because our system size is too small and we do not have enough energy levels in that region.

Finally, we note that the Landauer-Buttiker conductance discussed above applies to a wire that is connected to its leads by adiabatic, nonreflecting contacts. In the experiments by Steinberg and co-workers, ${ }^{7,8}$ the contacts from the finite wire to the two-dimensional electron gas are not perfectly adiabatic and will add contact resistance to any resistance discussed above.

\section{SUMMARY}

In summary, from our Hartree-Fock calculations, we have developed a picture of successive magnetic phases in the low density region of an inhomogeneous one-dimensional electron systems in a uniform magnetic field. The depleted electrons under the barrier first enter an antiferromagnetic phase; then, near depletion, part of the lowest density electrons become spin aligned and get isolated from the high density region outside the barrier by two antiferromagnetic regions sandwiching it. The final stage of depletion takes the form of successive expulsion of a single electron from the spinaligned region, resulting in successive periods of relative insensitivity of the spin-aligned electron density to $V_{g}$, followed by the sudden rearrangement due to the expulsion of one electron.

The most serious discrepancy between our calculations and the experimental results of Steinberg et al. is the form factor for momentum-conserved tunneling in the regime where there are of the order of four to ten electrons under the central gate. Our matrix elements have too much weight at large momenta. It is not clear what is the source of this discrepancy. However, it may be that potential fluctuations due to residual disorder are important. A small random potential due to charged impurities set back from the wire may have little effect on the mean free path for relatively high electron densities but could lead to strong backscattering and localization at very low densities, where the kinetic energy can be small and where the small value of $2 k_{F}$ permits backscattering from potential fluctuations of relatively long wavelength.

As mentioned earlier, calculations near the depletion of the upper band at a smaller $V_{g}$ give results similar to those obtained near the depletion of the lowest band. We find again a spin-aligned central region, sandwiched by antiferromagnetic regions on each side. A similar phenomenon of sudden expulsions of a single localized electron from the spinaligned parts is also observed. This is consistent with the experimental observation of similar localization behavior upon the depletion of the second subband. ${ }^{8}$ Small potential fluctuations due to impurities may again be important to explain the experimental results for momentum-dependent tunneling in this regime.

\section{ACKNOWLEDGMENTS}

We would like to thank Hadar Steiberg, Ophir Auslaender, Amir Yacoby, Yaroslav Tserkovnyak, and Greg Fiete for illuminating discussions. We have also benefited from discussions with Walter Hofstetter and Gergely Zarand when working on a related earlier project. This work is supported by NSF Grants No. DMR05-41988 and No. PHY06-46094.

\section{APPENDIX: SPIN CANTING IN THE HOMOGENEOUS SYSTEM}

Our calculations for the system with a barrier have been carried out using a restricted Hartree-Fock method, where canted spin states were not allowed. For a classical Heisenberg model in an applied uniform field $\vec{B}$, the antiferromagnetic state will spontaneously align itself so that the staggered spin component is perpendicular to the applied field and the individual spins will cant toward the direction of the

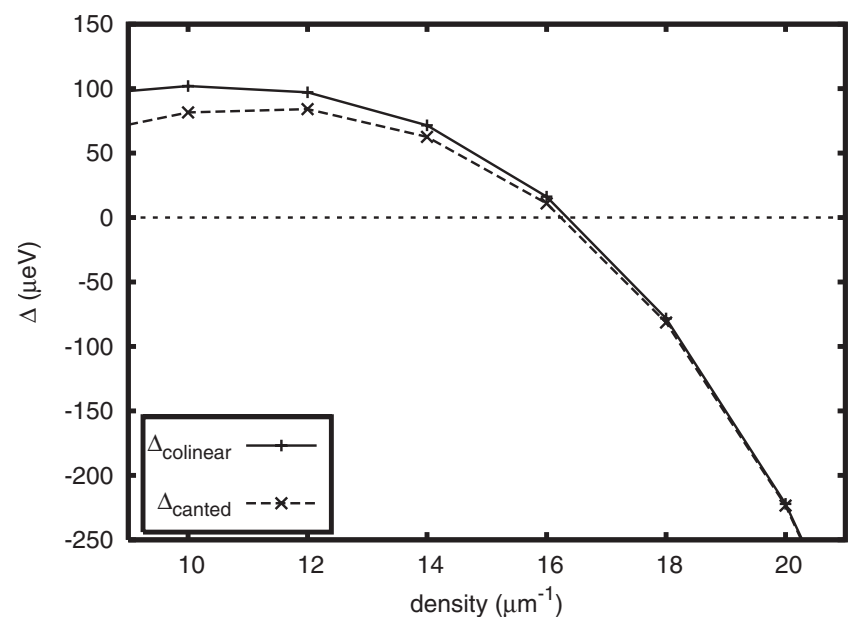

FIG. 13. The energy difference per electron, $\Delta$, between the antiferromagnetic and the fully spin-aligned solutions in an infinite uniform electron system as a function of electron density. Both systems are in a uniform magnetic field $B=1 \mathrm{~T}$. The dotted curve is the canted solution and the solid curve is the solution with spin collinear to $B$. 


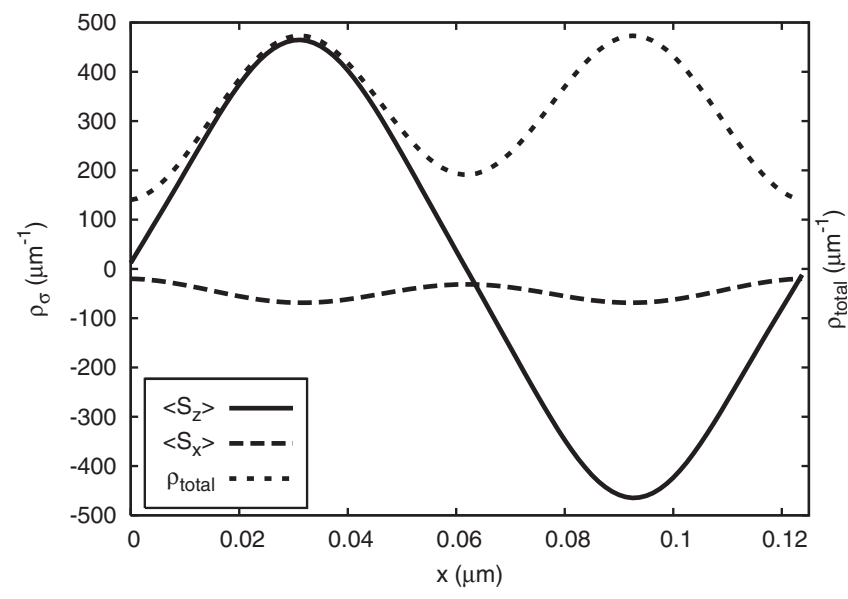

FIG. 14. The spin polarization in the $x$ and $z$ directions in the canted solution at density $\rho=16 \mu \mathrm{m}^{-1}$ right at the transition from the antiferromagnetic to the spin-aligned ground state. Due to the magnetic field in the $x$ direction, a small paramagnetic component is developed in addition to the dominant antiferromagnetic magnetization in the $z$ direction. Only a single unit cell, consisting of two electrons, are shown. The total density is also shown as the dotted line.

applied field. Hence, it may be asked whether allowing canting of the spins in a Hartree-Fock calculation would significantly change our results. To clarify this issue, we have carried out both restricted and unrestricted Hartree-Fock calculations on a uniform infinite electron system in a magnetic field. The interaction between electrons is the same as we used before, and the magnetic field is also $B=1 \mathrm{~T}$. As shown in Figs. 13 and 14, the effects of canting in terms of both the energy gains and the changes in spin density are small. Canting is only relevant to our zero-temperature calculation when the antiferromagnetic solution is in the ground state. Even near the transition density $\rho^{*}=16 \mu \mathrm{m}^{-1}$ from the antiferromagnetic ground state to the fully spin-aligned ground state, when canting is the greatest, the energy gained by allowing canting is only $2.64 \mu \mathrm{eV}$ per electron.

By extrapolating these results to the system with a barrier, we see that even if we allowed ten antiferromagnetic electrons on each side of the solution shown in Fig. 7 to cant, the total energy gain would be minuscule compared with the level spacing at the Fermi energy there. At $\rho^{*}$, in the homogeneous system, the magnetization parallel to the uniform magnetic field, in the canted state, is less than one-tenth of the antiferromagnetic magnetization perpendicular to it. Thus, our calculations for the homogeneous system suggest that allowing canting in the Hartree-Fock calculation of the inhomogeneous one-dimensional wire would not give qualitatively different results from our restricted calculations above.

We remark that for a homogeneous system in zero magnetic field, with the same electron-electron interaction as above, the onset of ferromagnetism in the Hartree-Fock approximation would occur at a density $\rho^{*} \approx 14 \mu \mathrm{m}^{-1}$.
${ }^{1}$ F. D. M. Haldane, J. Phys. C 14, 2585 (1981).

${ }^{2}$ J. Voit, Rep. Prog. Phys. 58, 977 (1995).

${ }^{3}$ K. A. Matveev, Phys. Rev. B 70, 245319 (2004).

${ }^{4}$ Y. Tserkovnyak, B. I. Halperin, O. M. Auslaender, and A. Yacoby, Phys. Rev. Lett. 89, 136805 (2002).

${ }^{5}$ G. A. Fiete, J. Qian, Y. Tserkovnyak, and B. I. Halperin, Phys. Rev. B 72, 045315 (2005).

${ }^{6}$ E. J. Mueller, Phys. Rev. B 72, 075322 (2005).

${ }^{7}$ O. M. Auslaender, O. M. Steinberg, A. Yacoby, Y. Tserkovnyak, B. I. Halperin, K. W. Baldwin, L. N. Pfeiffer, and K. W. West, Science 308, 88 (2005).
${ }^{8}$ H. Steinberg, O. M. Auslaender, A. Yacoby, J. Qian, G. A. Fiete, Y. Tserkovnyak, B. I. Halperin, K. W. Baldwin, L. N. Pfeiffer, and K. W. West, Phys. Rev. B 73, 113307 (2006).

${ }^{9}$ K. A. Matveev, Phys. Rev. Lett. 92, 106801 (2004).

${ }^{10}$ Y. Meir, K. Hirose, and N. S. Wingreen, Phys. Rev. Lett. 89, 196802 (2002).

${ }^{11}$ T. Rejec and Y. Meir, Nature (London) 442, 900 (2006).

${ }^{12} \mathrm{P}$. W. Anderson, Concepts in Solids (Addison-Wesley, Reading, MA, 1992).

${ }^{13}$ A. W. Overhauser, Phys. Rev. 128, 1437 (1962).

${ }^{14}$ E. Lieb and D. Mattis, Phys. Rev. 125, 164 (1962). 